\title{
A Chemical Perspective on Transcriptional Fidelity: Dominant Contributions of Sugar Integrity Revealed by Unlocked Nucleic Acids
}

\author{
Dr. Liang $\mathrm{Xu}^{\dagger}$, \\ Skaggs School of Pharmacy and Pharmaceutical Sciences, The University of California, San \\ Diego, La Jolla, California, 92093-0625, USA
}

Steven W. Plouffe ${ }^{\dagger}$, Skaggs School of Pharmacy and Pharmaceutical Sciences, The University of California, San Diego, La Jolla, California, 92093-0625, USA

\author{
Jenny Chong, \\ Skaggs School of Pharmacy and Pharmaceutical Sciences, The University of California, San \\ Diego, La Jolla, California, 92093-0625, USA
}

Dr. Prof. Jesper Wengel, and Nucleic Acid Center and Biomolecular Nanoscale Engineering Center, Department of Physics, Chemistry and Pharmacy, University of Southern Denmark, Campusvej 55, 5230 Odense M, Denmark

\section{Dr. Prof. Dong Wang}

Skaggs School of Pharmacy and Pharmaceutical Sciences, The University of California, San Diego, La Jolla, California, 92093-0625, USA

Dong Wang: dongwang@ucsd.edu

\section{Keywords}

synthetic nucleic acid analogues; RNA polymerase II; transcriptional fidelity; sugar integrity

\begin{abstract}
Nucleic acids (RNA and DNA) consist of three key structural moieties: the nucleobase, the sugar, and the phosphate group. A comprehensive understanding of how these intrinsic structural features are recognized by nucleic acid enzymes during gene expression is not only important for elucidating the chemical basis of the central dogma of biology, but also has many implications in synthetic biology and nucleic acid based therapeutics. ${ }^{[1-7]}$ Synthetic nucleic acid chemistry has now provided us with powerful tools to advance our understanding of the functional interplay between nucleic acids and nucleic acid enzymes, which cannot be achieved by conventional biological approaches. ${ }^{[1,8]}$
\end{abstract}

RNA Polymerase II (Pol II) is responsible for transcribing a DNA template into precursor messenger RNA in all eukaryotic cells. A key question is how Pol II recognizes the structural moieties of nucleic acids and how it maintains high transcriptional fidelity. Previous studies mainly focused on understanding the contributions of the nucleotide's peripheral functional groups (the nucleobase and $2^{\prime}-\mathrm{OH}$ group) to Pol II transcriptional

\footnotetext{
Correspondence to: Dong Wang, dongwang@ucsd. edu.

${ }^{\dagger}$ These authors contributed equally to this work.

Supporting information for this article is available on the WWW under http://www.angewandte.org or from the author.
} 
fidelity. ${ }^{[9-13]}$ However, the contribution of the central structural moiety (the sugar backbone) is unclear. It is worth noting that nature has evolved to select cyclic ribose as the sugar backbone for both RNA and DNA, and modern nucleic acid enzymes, such as Pol II, were adapted for this sugar backbone during evolution. This raises several intriguing questions: how important is this intact sugar backbone in contributing to Pol II substrate recognition and transcriptional fidelity? Do modern nucleic acid enzymes, such as Pol II, have a "built-in" capacity to recognize and select for nucleotide and nucleic acids with an intact sugar backbone?

To address these questions, we employed a synthetic chemical biology approach by comparing Pol II transcription of a canonical, "wild type" nucleotide to that of a "sugar backbone mutant" nucleotide analogue (Figure 1). This synthetic "sugar backbone mutant", termed Unlocked Nucleic Acid (UNA), ${ }^{[14-17]}$ contains all peripheral functional groups of the natural nucleotide except it has a disrupted ribose ring (Figure 1a). ${ }^{[14]}$ Importantly, the UNA residues remain able to form Watson-Crick base-pairing with RNA or DNA strands without significant disruption to the duplex structure. ${ }^{[18]}$ Here we systematically and quantitatively dissected the contribution of the sugar backbone to Pol II substrate incorporation, elongation, and the three key checkpoint steps of transcriptional fidelity. This knowledge is not only important for elucidating the molecular basis of sugar recognition by Pol II, but also provides insight into understanding the molecular basis of genetic information storage and transfer during evolution.

To understand the effect of the disrupted sugar backbone on Pol II substrate incorporation, we focused on dissecting the contributions of two components: the incoming substrate and the RNA primer. Here, two critical enzymatically kinetic parameters were determined: substrate specificity constant $\left(k_{\mathrm{pol}} / K_{\mathrm{d} \text {,app }}\right)$, which is derived from the rate of polymerization $\left(k_{\mathrm{pol}}\right)$ and apparent substrate dissociation constant $\left(K_{\mathrm{d}, \mathrm{app}}\right)$, and discrimination calculated from the ratio of the $k_{\mathrm{pol}} / K_{\mathrm{d} \text {,app }}$ values of substrate A over substrate $\mathrm{B}\left(\left(k_{\mathrm{pol}} / K_{\mathrm{d}, \mathrm{app}}\right)_{\mathrm{A}} /\left(k_{\mathrm{pol}} /\right.\right.$ $\left.\left.K_{\mathrm{d} \text {,app }}\right)_{\mathrm{B}}\right)$. Discrimination is a quantitative measure of the enzyme's discrimination power of two substrates. To study the effect of a disrupted sugar in the substrate, we compared the nucleotide incorporation efficiency of canonical ATP substrate and unlocked adenosine triphosphate analogue (denote as $\boldsymbol{a} \mathrm{TP}$ ) to Pol II elongation complex with the scaffold I containing a 10nt RNA primer with an A at 3'-RNA terminus (termed 10A, Figure S1a). Strikingly, the nucleotide incorporation of $\boldsymbol{a}$ TP substrate is substantially slower than that of ATP (Figure 2a), and Pol II exhibits a $\sim 10^{7}$-fold discrimination of ATP over $\boldsymbol{a}$ TP $\left(\left(k_{\mathrm{pol}} /\right.\right.$ $\left.\left.K_{\mathrm{d}, \text { app }}\right)_{\mathrm{ATP}} /\left(k_{\mathrm{pol}} / K_{\mathrm{d} \text {,app }}\right)_{\boldsymbol{a T P}}\right)$ as revealed by pre-steady state single turn-over incorporation assays (Figure 2c, Table S1), even though both substrates share the same nucleobase, sugar hydroxyl, and triphosphate functional groups. This strong discrimination largely results from the difference in catalytic rates $\left(\sim 10^{6}\right.$-fold) rather than a difference in the substrate affinity ( 10 -fold) (Figure S2). It is unexpected that the contribution of the sugar backbone $\left(\sim 10^{7}\right.$ fold in $k_{\mathrm{pol}} / K_{\mathrm{d} \text {,app }}$ ) is $\sim 100$-fold stronger than that of nucleobase pairing (matched A:dT pair versus mismatched $\mathrm{U}: \mathrm{dT}$ measured by $\left.\left(k_{\mathrm{pol}} / K_{\mathrm{d}, \mathrm{app}}\right)_{\mathrm{ATP}} /\left(k_{\mathrm{pol}} / K_{\mathrm{d}, \mathrm{app}}\right)_{\mathrm{UTP}}\right)$ and $103-104$ stronger than that of sugar 2'-OH interactions (NTP versus dNTP) ${ }^{9,11,19]}$ This result reveals that the integrity of the substrate's sugar backbone plays a dominant role in ensuring efficient Pol II substrate incorporation.

To investigate the role of sugar integrity of the RNA primer, we compared nucleotide incorporation to Pol II elongation complexes with either a scaffold containing a canonical RNA primer (10A) or a scaffold containing a RNA primer with an unlocked adenosine $(\boldsymbol{a})$ substitution at the $3^{\prime}$-RNA terminus $\left(10 a_{\mathrm{n}}, \mathrm{n}\right.$ referred the $3^{\prime}$-RNA terminus position) (Figure 2a). We found that AMP incorporation to $10 a_{n}$ is substantially slower than the incorporation to scaffold $10 \mathrm{~A}$, with a $\sim\left(2 \times 10^{5}\right)$-fold reduction of $k_{\mathrm{pol}} / K_{\mathrm{d} \text {,app }}$ value (Figure $2 \mathrm{c}$ and Table S1). This reveals an important role for sugar integrity at the $3^{\prime}$-RNA terminus for efficient 
nucleotide incorporation. No $a \mathrm{MP}$ incorporation (unlocked adenosine monophosphate) to the $10 a_{\mathrm{n}}$ primer was observed even after extensive incubation periods (Figure 2a), suggesting that the disrupted sugar backbone of both substrate and 3'-RNA terminal residue together completely abolishes Pol II transcription.

To further test the effect of UNA incorporation in the RNA primer on Pol II elongation, we systematically designed primers containing a site-specific UNA residue at either the $\mathrm{n}, \mathrm{n}-1$, or $\mathrm{n}-2$ positions (termed $10 a_{\mathrm{n}}, 10 \boldsymbol{g}_{\mathrm{n}-1}$, or $10 \mathrm{~g}_{\mathrm{n}-2}$, respectively, Figure $2 \mathrm{~b}$ ). These scaffolds allowed us to compare the effect of the disrupted sugar backbone at different positions along the RNA primer on Pol II elongation. We observed UNA at the $3^{\prime}$-RNA terminus $\left(10 a_{\mathrm{n}}\right)$ greatly inhibits Pol II elongation, whereas much less inhibitory effects on Pol II elongation with the $10 g_{\mathrm{n}-1}$ and $10 g_{\mathrm{n}-2}$ (Figure $2 \mathrm{~b}$ ). The $k_{\mathrm{pol}} / K_{\mathrm{d} \text {,app }}$ value of nucleotide incorporation of ATP to $10 g_{\mathrm{n}-2}$ is only $\sim 5$-fold less than that to the $10 \mathrm{~A}$ scaffold, compared to a $\left(2 \times 10^{5}\right)$-fold reduction in specificity of nucleotide incorporation to $10 a_{\mathrm{n}}$ (Figure $2 \mathrm{~d}$ and Table $\mathrm{S} 1$ ). These results indicate that the effect of RNA primer sugar integrity on Pol II transcription is position-dependent, and the inhibitory effect on Pol II transcription is greatly abolished as the UNA residue moves further upstream from the $3^{\prime}$-RNA terminus.

Pol II transcriptional fidelity is maintained by three checkpoint steps: (1) specific nucleotide selection and incorporation at the $3^{\prime}$-RNA terminus, (2) discrimination between matched and mismatched nucleotides of the $3^{\prime}$-RNA terminus during elongation, and (3) proofreading activity via preferential cleavage of misincorporated nucleotides. ${ }^{[8,11]} \mathrm{We}$ assembled five active RNA Pol II elongation complexes with either an unmodified RNA primer or one containing a site-specific UNA modification (Figure S1, scaffolds I and II). This system allowed us to systematically and quantitatively measure the contributions of sugar integrity at each of the three Pol II transcriptional fidelity checkpoint steps.

To measure the contribution of sugar integrity on the first Pol II transcriptional fidelity checkpoint step (nucleotide selection and incorporation), we measured the kinetic parameters for matched ATP, mismatched UTP, sugar-disrupted $\boldsymbol{a}$ TP, and mismatched and sugar-disrupted $\boldsymbol{u} \mathrm{TP}$ incorporation, respectively (Figure 3a, Figure S2, Table 1).

Comparison of the $k_{\mathrm{pol}} / K_{\mathrm{d} \text {,app }}$ values among the four substrates leads to two very striking results (Figure 3a, Table 1$)$. First, Pol II's discrimination for sugar integrity $\left(\left(k_{\mathrm{pol}} /\right.\right.$ $\left.K_{\mathrm{d} \text {,app }}\right)_{\mathrm{ATP}} /\left(k_{\mathrm{pol}} / K_{\mathrm{d} \text {,app }}\right)_{\boldsymbol{a}} \mathrm{TP}, \sim 10^{7}$-fold $)$ is $\sim 100$-fold higher than its nucleobase discrimination $\left(\left(k_{\mathrm{pol}} / K_{\mathrm{d} \text {,app }}\right)_{\mathrm{ATP}} /\left(k_{\mathrm{pol}} / K_{\mathrm{d} \text {,app }}\right)_{\mathrm{UTP}}, \sim 10^{5}\right.$-fold $)$. Second, Pol II's nucleobase discrimination of ATP over a mismatched UTP is completely abolished following the replacement of the canonical ribose with a UNA sugar (dropped from $\sim 10^{5}$-fold to $\sim 1$ ). This clearly indicates that the disruption of the sugar backbone integrity not only greatly reduces Pol II transcriptional efficiency but also abolishes nucleobase recognition during incorporation, indicating that sugar backbone integrity is a prerequisite for correct nucleotide selection in Pol II transcription.

To investigate the effect of a disrupted sugar backbone at the second checkpoint step (transcript extension), we assembled four Pol II elongation complexes with scaffold II containing the following RNA:DNA pairings at the $3^{\prime}$-RNA terminus: A:dT, U:dT, $\boldsymbol{a}: \mathrm{dT}$, and $\boldsymbol{u}$ :dT respectively (termed $11 \mathrm{~A}, 11 \mathrm{U}, 11 \boldsymbol{a}$, and $11 \boldsymbol{u}$, Figure $3 \mathrm{~b}$ and Figure S1). These four Scaffold II mimick four potential product states of Pol II elongation of scaffold I after the first checkpoint step (Figure 3b). This approach allowed us to evaluate the influence of both sugar integrity and base pairing at the $3^{\prime}$-RNA terminus of the primer on incorporation of the next nucleotide. We measured the specificity constants of nucleotide incorporation of CTP to all four Pol II elongation complexes. Consistently, the efficiency of nucleotide incorporation following the correct A:dT base pair is $\sim 10^{5}$-fold higher than that following 
the mismatched base pair U:dT (Figure 3b, Figure S3 and Table 1). ${ }^{[8]}$ Strikingly, in the absence of sugar integrity, Pol II's ability to discriminate between a terminal matched pair over a terminal mismatched pair was significantly reduced from $10^{5}$-fold (extension of A:dT over U:dT) to 6.2-fold (extension of $\boldsymbol{a}: \mathrm{dT}$ over $\boldsymbol{u}: \mathrm{dT}$ ) (Figure 3b, Figure S3 and Table 1). These results suggest that sugar backbone integrity is also a dominant factor in controlling the second checkpoint step of Pol II transcriptional fidelity.

Finally, to investigate how the presence of incorporated UNA in the RNA primer affects the third transcriptional fidelity checkpoint step (proofreading), we measured TFIIS-simulated cleavage rates for four Pol II elongation complexes containing the following RNA:DNA pairs at the 3'-RNA terminus: A:dT, U:dT, $\boldsymbol{a}$ :dT, and $\boldsymbol{u}$ :dT (scaffold II 11A, 11U, $11 \boldsymbol{a}$, and $11 u$, Figure $3 \mathrm{c}$ and Figure S1). The cleavage rate for 11A with a matched A:dT pair is about 12 -fold slower than that of $11 \mathrm{U}$ with a mismatched U:dT pair (Figure $3 \mathrm{c}$ and Table 1). ${ }^{[8]}$ Intriguingly, we found that replacing the canonical sugar with unlocked sugar also resulted in a large increase ( 14-fold) in cleavage rate for $11 \boldsymbol{a}$ in comparison with that for $11 \mathrm{~A}$, even if the nucleobase was correctly matched to the dT template. Thus, Pol II is unable to discriminate between a matched end $(11 a: \mathrm{dT})$ and a mismatched end $(11 \boldsymbol{u}: \mathrm{dT})$ in the absence of terminal sugar integrity. Notably, the sugar substitution did not change the corresponding cleavage pattern (Figure S4). Taken together, UNA sugar substitution at the 3'-RNA terminus ablates the Pol II proofreading activity.

In summary, we systematically dissected the contribution of the nucleic acid sugar backbone to Pol II transcription. Surprisingly, we revealed that during Pol II transcription, the contribution of the sugar backbone for enzymatic efficiency is $10^{3}-10^{4}$ stronger than that of the $2^{\prime}-\mathrm{OH}$ group on the ribose and $\sim 100$-fold stronger than that of base pairing. Even more strikingly, we found that sugar integrity is a dominant factor in controlling all three checkpoint steps of Pol II transcriptional fidelity. These results provide novel insights into the molecular basis of sugar backbone recognition by Pol II, which has been underappreciated. Sugar backbone integrity is a prerequisite for ensuring correct nucleotide selection, incorporation, and extension in Pol II transcription as revealed by nucleobase discrimination being completely abolished following replacement with an unlocked sugar. The nucleotide's peripheral functional groups per se are insufficient for efficient Pol II transcription. Rather, correct spatial arrangement of the functional groups, which are guided by the sugar backbone, is key to maintaining high Pol II catalytic activity and fidelity. This study may also provide some insight into understanding how nucleic acid enzymes evolved the "built-in" capability to recognize key structural features of nucleic acids in general.

\section{Supplementary Material}

Refer to Web version on PubMed Central for supplementary material.

\section{Acknowledgments}

D.W. acknowledges the NIH (GM102362), Kimmel Scholar Award from the Sidney Kimmel Foundation for Cancer Research, and start-up funds from Skaggs School of Pharmacy and Pharmaceutical Sciences, UCSD. S.W.P. was supported in part by the UCSD Graduate Training Program in Cellular and Molecular Pharmacology training grant (T32 GM007752).

\section{References}

1. Benner SA, Sismour AM. Nat Rev Genet. 2005; 6:533-543. [PubMed: 15995697]

2. Opalinska JB, Gewirtz AM. Nat Rev Drug Discov. 2002; 1:503-514. [PubMed: 12120257]

3. Benner SA. Acc Chem Res. 2004; 37:784-797. [PubMed: 15491125]

4. Benner SA. Science. 2004; 306:625-626. [PubMed: 15499002] 
5. Krueger AT, Kool ET. Chem Biol. 2009; 16:242-248. [PubMed: 19318205]

6. Carell T, Brandmayr C, Hienzsch A, Muller M, Pearson D, Reiter V, Thoma I, Thumbs P, Wagner M. Angew Chem. 2012; 124:7220-7242.Angew Chem Int Ed Engl. 2012; 51:7110-7131. [PubMed: 22744788]

7. Pinheiro VB, Holliger P. Curr Opin Chem Biol. 2012; 16:245-252. [PubMed: 22704981]

8. Kellinger MW, Ulrich S, Chong J, Kool ET, Wang D. J Am Chem Soc. 2012; 134:8231-8240. [PubMed: 22509745]

9. Kaplan CD, Larsson KM, Kornberg RD. Mol Cell. 2008; 30:547-556. [PubMed: 18538653]

10. Kireeva ML, Nedialkov YA, Cremona GH, Purtov YA, Lubkowska L, Malagon F, Burton ZF, Strathern JN, Kashlev M. Mol Cell. 2008; 30:557-566. [PubMed: 18538654]

11. Yuzenkova Y, Bochkareva A, Tadigotla VR, Roghanian M, Zorov S, Severinov K, Zenkin N. BMC Biol. 2010; 8:54. [PubMed: 20459653]

12. Erie DA, Hajiseyedjavadi O, Young MC, von Hippel PH. Science. 1993; 262:867-873. [PubMed: 8235608]

13. Su Zhang DW. Isr J Chem. 2013; 53:442-449.

14. Langkjaer N, Pasternak A, Wengel J. Bioorg Med Chem. 2009; 17:5420-5425. [PubMed: 19604699]

15. Werk D, Wengel J, Wengel SL, Grunert HP, Zeichhardt H, Kurreck J. FEBS Letters. 2010; 584:591-598. [PubMed: 20005874]

16. Laursen MB, Pakula MM, Gao S, Fluiter K, Mook OR, Baas F, Langklaer N, Wengel SL, Wengel J, Kjems J, Bramsen JB. Mol Biosyst. 2010; 6:862-870. [PubMed: 20567772]

17. Campbell MA, Wengel J. Chem Soc Rev. 2011; 40:5680-5689. [PubMed: 21556437]

18. Pasternak A, Wengel J. Nucleic Acids Res. 2010; 38:6697-6706. [PubMed: 20562222]

19. Wang D, Bushnell DA, Westover KD, Kaplan CD, Kornberg RD. Cell. 2006; 127:941-954. [PubMed: 17129781] 
a

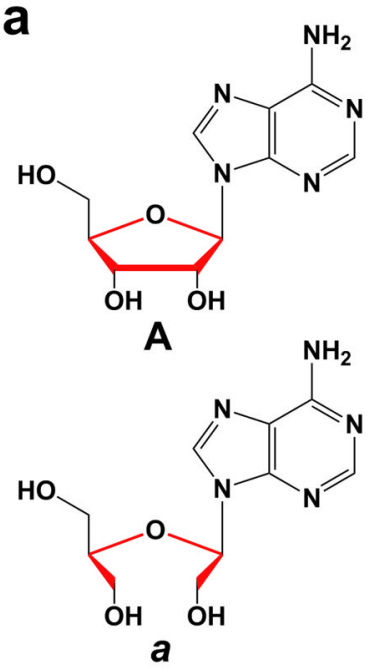

b

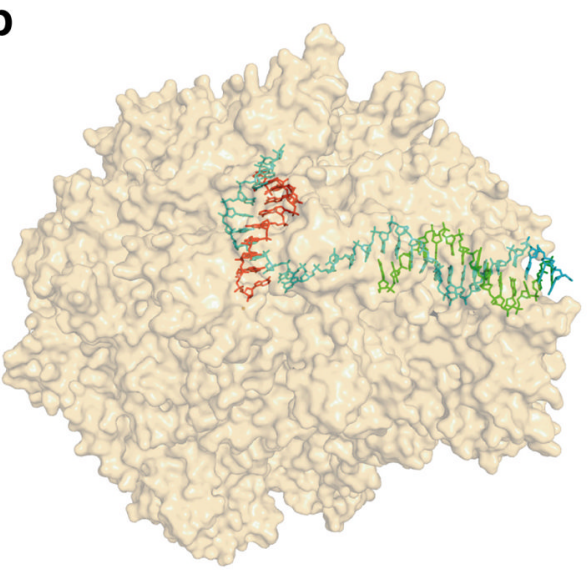

Figure 1.

Canonical and unlocked nucleosides used in this study. a) Structures of adenosine (A) and unlocked adenosine (denoted as $\boldsymbol{a}$ ). The sugar backbones are highlighted in red. b) Structure of Pol II elongation complex (PDB: 2E2H). ${ }^{[19]}$ Pol II, RNA, template DNA, and nontemplate DNA are shown in tan, red, cyan, and green, respectively. 

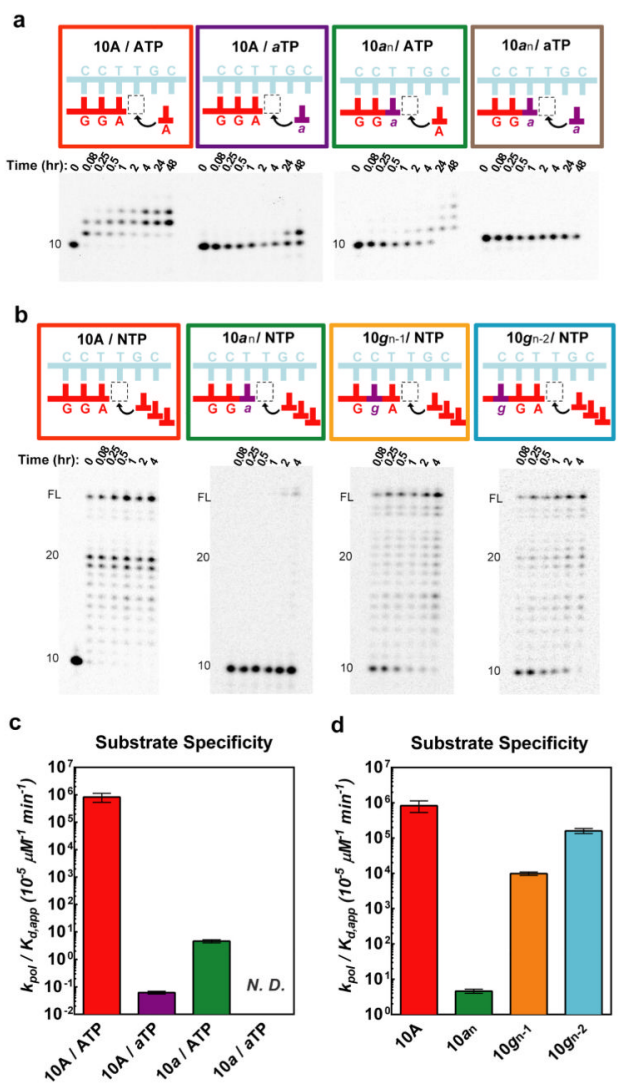

Figure 2.

Sugar backbone integrity plays an important role in ensuring efficient Pol II transcription. a) ATP or $\boldsymbol{a}$ TP incorporation to $10 \mathrm{~A}$ or $10 \boldsymbol{a}_{n}$ scaffold by Pol II. b) Effects of an unlocked nucleotide substitution at the RNA primer on Pol II elongation. All NTP, ATP and $\boldsymbol{a}$ TP are 1 mM. c) Loss of sugar backbone integrity greatly reduces the substrate specificity constants. d) Pol II elongation efficiency is recovered as the unlocked nucleoside is moved toward the upstream positions. 


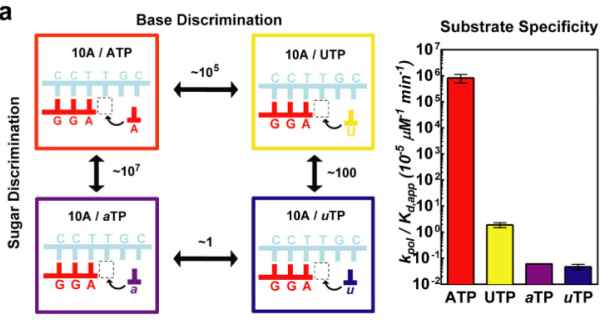

b
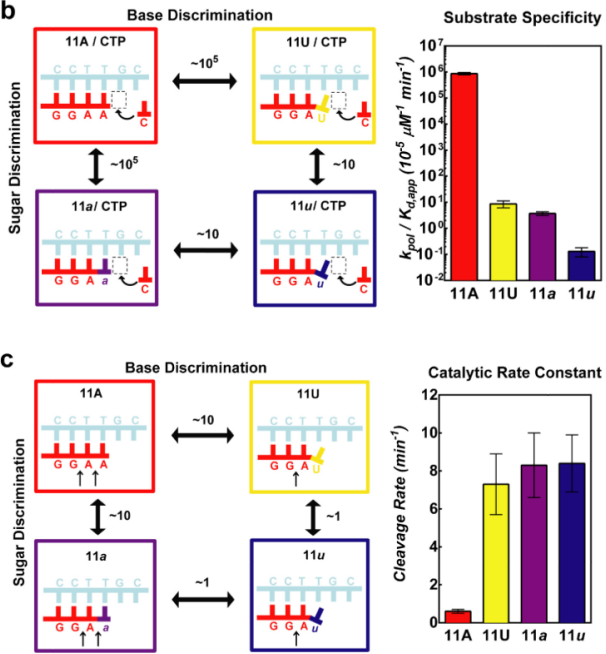

Figure 3.

Sugar backbone integrity plays a dominant role in Pol II transcriptional fidelity in all three fidelity checkpoint steps. a) Loss of sugar backbone integrity completely abolishes the nucleobase discrimination in the first checkpoint step. b) Loss of sugar backbone integrity significantly abolishes the primer nucleobase discrimination in the second checkpoint step. c) Loss of sugar integrity abolishes the difference of TFIIS-mediated cleavage for matched over mismatched $3^{\prime}$-RNA:DNA terminus in the third checkpoint step. 


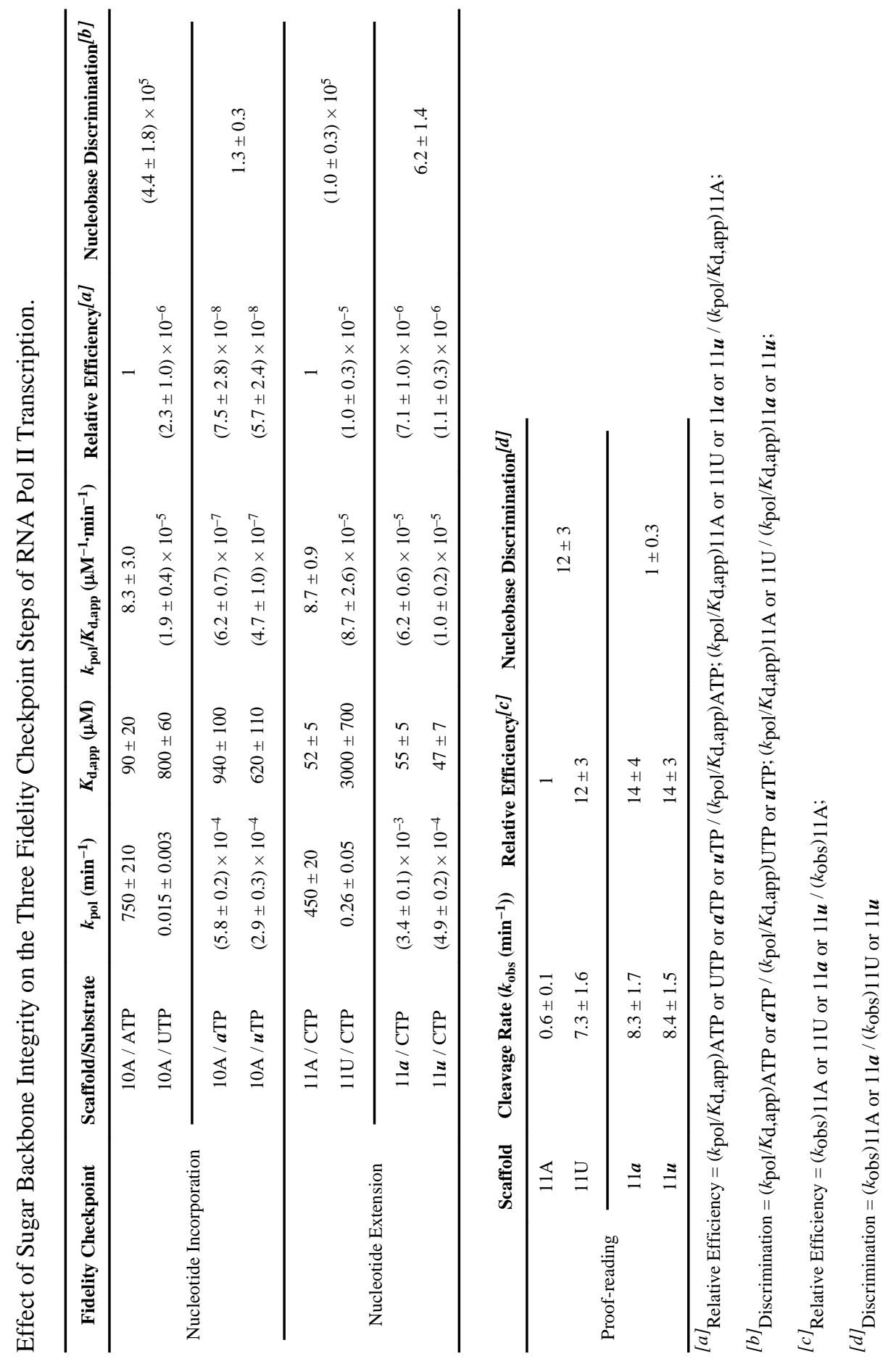

